\title{
An advertisement analysis of alcohol products in popular women's magazines
}

\author{
M Dottington Fullwood ${ }^{1 *}$, Jennifer Mongiovi ${ }^{2}$, Grace Hillyer ${ }^{2}$, Corey H. Basch ${ }^{3}$, Danna Ethan ${ }^{4}$ and Rodney Hammond ${ }^{3,5}$ \\ ${ }^{1}$ Department of Health and Behavior Studies, Teachers College, Columbia University, New York, USA \\ ${ }^{2}$ Department of Epidemiology, Mailman School of Public Health, Columbia University, New York, USA \\ ${ }^{3}$ Department of Public Health, William Paterson University, NJ, USA \\ ${ }^{4}$ Department of Health Sciences, Lehman College, The City University of New York, New York, USA \\ ${ }^{5}$ Department of Health and Nutrition Sciences, Montclair State University, 1 Normal Ave, Montclair, NJ, USA
}

\section{Introduction}

In the United States, the use of alcohol is largely a social activity and the patterns and trends of consumption vary by gender, and cultural and ethnic group [1,2]. Recent national survey data demonstrate that, among women between the ages of 18 and 44 years, $53.6 \%$ had consumed any alcohol in the past 30 days and use was highest among 21-24 year old, non-Hispanic white women with a college degree. The misuse of alcohol, for example, heavy drinking defined as $>14$ drinks per week for males and $>7$ for women or binge drinking in which 5 drinks for men and 4 drinks for women are consumed in 2 hours or less [3], has important health ramifications. Alcohol misuse is related to over 200 diseases including liver cirrhosis and cancer and, if used during pregnancy, can lead to fetal alcohol spectrum disorders [4]. Misuse of alcohol is also a major contributor to injury-related health conditions, motor vehicle crashes, and violence [5]. The economic burden of excessive alcohol use, reported at $\$ 223.5$ billion in 2006 , is a direct result of premature death, disability, lost workplace productivity, and healthcare expenses, making alcohol consumption a critically important public health issue [6].

The Federal Trade Commission (FTC) regulates alcohol advertising to ensure that what is presented to the public is truthful and non-deceptive. The FTC additionally encourages the alcohol industry to self-regulate with regard to the placement and content of alcohol advertisements, particularly when advertisements may be viewed by teenagers [7]. Over the years, the intended audiences of alcohol advertising campaigns have changed with drinks such as beer and whiskey marketed primarily to men and other fruitier, feminine, "easy to drink" sweet alcohol products to women [8]. It has been argued that gender stereotyping in alcohol advertising exploits sexism in our society and attempts to link products with attractiveness to the opposite gender [8].

The current study evaluated the number and content of alcohol product (hard liquor and beer) advertisements in popular U.S. interest or lifestyle magazines for women. Themes of the advertisements were characterized and differences in alcohol product advertisement placement in magazines targeting non-Hispanic white and Black and Latina audiences were evaluated.

\section{Materials and methods}

We conducted a descriptive analysis of alcohol advertisements in 14 popular U.S. magazines marketed to women in the U.S. These magazines included Allure, Cosmopolitan, Cosmopolitan for Latinas, Ebony, Elle, Essence, Girl's Life, Glamour, Jet, Latina, Marie Claire, Seventeen, Teen Vogue, Vogue. A total of 98 issues published between January and August, 2014 were reviewed. The combined readership of these magazines is nearly 80 million female readers with a mean readership age of 37.5 years and a median household income of $\$ 62,087$ [9-21]. Of the magazines reviewed, five were specifically marketed to a Black or Latina audience: Cosmopolitan for Latinas, Ebony, Essence, Jet, Latina.

Paid advertisements for alcohol products (hard liquor and beer) throughout the issue as well as those on the back cover were evaluated. Advertisements highlighted as "editor's picks" or featured in editorials or articles were excluded from this analysis. Characteristics of the magazine readership were assessed and included the median age and household income of the readership. For each advertisement we assessed whether a warning related to the use of alcohol was present and if healthy-appearing models were used in the advertisement. Advertisements were categorized by the following themes: 1) partying 2) having fun with friends 3) being cool 4) enjoyment, 5) happiness, and 6) having a free spirit. The theme of "partying" was defined as a scene involving alcohol and a large group of individuals at a special event, whereas "fun with friends" portrayed a more intimate group of fewer individuals in a casual atmosphere. "Being cool" was depicted by an image conveying a sense of status by the product's consumer in the advertisement. "Enjoyment" focused on the pleasure of drinking the product as opposed to the implication of happiness conveyed in a scene in which drinking was not necessarily shown, but alcohol was present and models exhibited a positive mental state. Finally, "having a free spirit" was defined as an advertisement depicting a non-conformist type of individual.

Descriptive analyses included mean readership $\leq 38$ years age of dichotomized age vs. as $>38$ years $\leq \$$ and 62,087 median vs. $>$ household. \$62,087 income Frequency distributions for advertisement characteristics (warnings present, models depicted in the advertisement

Correspondence to: M. Dottington Fullwood, MS, 125 W 120th St. Box 114, New York, NY 10027, Tel: (212)-678-6607; E-mail: Fullwood@tc.columbia.edu

Received: January 21, 2016; Accepted: February 22, 2016; Published: February 25, 2016 
and advertisement themes). All analyses were performed using IBM SPSS (version 22). The Institutional Review Boards at Teachers College, William Paterson University, Columbia University, Montclair State University, and Lehman College do not review studies that do not involve human subjects.

\section{Results}

Overall, there were 16 advertisements in the 98 issues of the 14 magazines reviewed. The alcohol advertisements were distributed equitably in magazines by the median age and household annual income of the readership (Table 1). Warnings were displayed in only $62.5 \%$ of advertisements and models with the products were seen in $37.5 \%$. "Being cool" was the dominant theme of the advertisements. Of the 16 advertisements, $14(87.5 \%)$ were found in magazines for the non-Hispanic White audience and $2(12.5 \%)$ in magazines for the Black and Latina markets. Compared to the alcohol advertisements in the magazines for Black and Latina women, the advertisements for the non-Hispanic White market were more often displayed in magazines for the $\leq 38$ younger vs.. $>38$ (years) and more affluent households $(>\$ 62,000$ vs. $\leq \$ 62,000)$. Fewer advertisements in the non -Hispanic White magazines showed warnings (57.1\%). The dominant themes in the non-Hispanic White magazine alcohol advertisements were having fun with friends and being cool as opposed to partying and happiness in the 2 advertisements in magazines marketed to Black and Latina women.

\section{Discussion}

The findings of this study are interesting for several reasons. First, it was determined that hard liquor advertisements were found more frequently than any other type of alcohol beverages. While the annual

Table 1. Characteristics of magazine readership and advertisements by alcohol product type $(n=16)$.

\begin{tabular}{|c|c|c|c|}
\hline & \multirow[b]{2}{*}{$\begin{array}{c}\text { Total alcohol }^{\text {a }} \\
\text { advertisement }^{\text {advel6 }}\end{array}$} & \multicolumn{2}{|c|}{ Audience } \\
\hline & & $\begin{array}{c}\text { Non-Hispanic } \\
\text { White } \\
\mathrm{N}=\mathbf{1 4}(\mathbf{8 7 . 5 \% )})\end{array}$ & $\begin{array}{l}\text { Black/Latina }^{\mathrm{b}} \\
\mathrm{N}=2(12.5 \%)\end{array}$ \\
\hline \multicolumn{4}{|l|}{ Readership characteristics } \\
\hline \multicolumn{4}{|l|}{ Median age (years) } \\
\hline$\leq 38$ & $9(56.3)$ & $9(100.0)$ & $0(0.0)$ \\
\hline$>38$ & $7(43.7)$ & $5(71.4)$ & $2(28.6)$ \\
\hline \multicolumn{4}{|l|}{ Median household income } \\
\hline$\leq \$ 62,000$ & $8(50.0)$ & $6(42.9)$ & $2(100.0)$ \\
\hline$>\$ 62,000$ & $8(50.0)$ & $8(57.1)$ & $0(0.0)$ \\
\hline \multicolumn{4}{|l|}{ Advertisement characteristics } \\
\hline Warnings present advertisement & $10(62.5)$ & $8(57.1)$ & $2(100.0)$ \\
\hline \multicolumn{4}{|l|}{ Themes in advertisement ${ }^{\mathrm{c}}$} \\
\hline Partying & $4(25.0)$ & $3(21.4)$ & $1(50.0)$ \\
\hline Fun with friends & $9(56.3)$ & $9(64.3)$ & $0(0.0)$ \\
\hline Being "cool" & $11(68.8)$ & $11(78.6)$ & $0(0.0)$ \\
\hline Enjoyment & $2(12.5)$ & $2(14.3)$ & $0(0.0)$ \\
\hline Happiness & $4(25.0)$ & $3(21.4)$ & $1(50.0)$ \\
\hline Free spirit & $0(0.0)$ & $0(0.0)$ & $0(0.0)$ \\
\hline Healthy models in advertisement & $6(37.5)$ & $5(35.7)$ & $1(50.0)$ \\
\hline
\end{tabular}

revenue is in the billions per year for both beer and hard liquor, it was hard liquor that was more likely to appear in advertisements [22,23].

Second, the majority of alcohol advertisements were found in magazines marketed toward non-Hispanic White audiences. While the health consequences of developing alcohol-related liver disease are greater and there are higher rates of binge drinking in Hispanics and Blacks as compared to Whites [24], advertisements for alcohol were not more prevalent in magazines geared toward these audiences. Nevertheless, health warnings in advertisements could help curb the potential life lost and unintentional injuries.

\section{Conclusion}

The adverse consequences associated with alcohol overconsumption can result in a disruption between friends, family, and social support [25]. Overall, the findings of this study indicate that alcohol advertisements were not abundant in the magazines included in this sample. Although not found in this study, research suggests that minority communities are more likely to be the target of alcohol advertisements [26]. Information and tactics like customized social marketing strategies are needed to counterbalance distinct advertising techniques that entice women, and Black and Latina women in particular are needed.

This study is limited by the cross-sectional design and the small sample size. However, it contributes to the literature on this subject by including a wide range of magazines including both those marketed to a Black and/or Latina audience. Future research can benefit from inclusion of additional magazines, over a greater period of time, with analysis of additional themes. Additional studies can also focus on the extent to which alcohol advertisements activate a response to initiate alcohol consumption.

\section{Funding}

This study was unfunded. The authors have no financial conflicts to disclose.

\section{References}

1. Module 10H: Ethnicity, culture and alcohol. NIAAA: Social work education for the prevention and treatment of alcohol use disorders (2005) National Institute on Alcohol Abuse and Alcoholism.

2. Social and cultural aspects of drinking: A report to the European Commission (1998) Social Issues Research Centre (Vol. 2015). Oxford, England.

3. Alcohol and your health: Alcohol facts and statistics (2015) National Institute on Alcohol Abuse and Alcoholism.

4. Alcohol and your health: Drinking levels defined (2015) National Institute on Alcohol Abuse and Alcoholism.

5. Tan CH, Denny CH, Cheal NE, Sniezek JE, Kanny D (2015) Alcohol use and binge drinking among women of childbearing age - United States, 2011-2013. MMWR Morb Mortal Wkly Rep 64: 1042-1046.

6. Excessive drinking costs U.S. $\$ 223.5$ billion (2014) Centers for Disease Control and Prevention. CDC Features: Data \& Statistics.

7. Evans JM, Krainsky E, Fentonmiller K, Brady C, Yoeli E et al. (2014) Self-regulation in the alcohol industry: Report to the Federal Trade Commission. Washington D.C.: Federal Trade Commission.

8. Clement K, Astrich J, Graham M, Cansler S (2010) How gender affects approaches in alcohol advertising.

9. Allure Media Kit (2015) Conde Nast.

10. Glamour Media Kit (2015) Conde Nast.

11. Teen Vogue Media Kit (2015) Conde Nast. 
12. Vogue Media Kit (2015) Conde Nast.

13. Latina Media Kit (2015) Direct Action Media.

14. Girls Life Magazine (2015) Echo Media.

15. Cosmopolitan Media Kit (2015) Hearst Corporation.

16. Elle Media Kit (2015) Hearst Corporation.

17. Marie Claire Media Kit (2015) Hearst Corporation.

18. Seventeen Media Kit (2015) Hearst Corporation.

19. Ebony Media Kit (2015) Johnson Publishing Company.

20. Jet Magazine Micro Media Kit (2015) Johnson Publishing Company.
21. Essence Media Kit (2015) Time Inc.

22. National beer sales and production data (2016) Brewers Association.

23. Economic contributions of the distilled spirits industry (2016) Distilled Spirits Council of the United States.

24. Minority health and health disparities, National Institute on Alcohol Abuse and Alcoholism.

25. Palamar JJ, Fenstermaker M, Kamboukos D, Ompad DC, Cleland CM, et al. (2014) Adverse psychosocial outcomes associated with drug use among US high school seniors: a comparison of alcohol and marijuana. Am J Drug Alcohol Abuse 40: 438446. [Crossref]

26. Jones-Webb R, Karriker-Jaffe KJ (2013) Neighborhood disadvantage, high alcohol content beverage consumption, drinking norms, and drinking consequences: a mediation analysis. J Urban Health 90: 667-684. [Crossref]

Copyright: ( 2016 Dottington Fullwood M. This is an open-access article distributed under the terms of the Creative Commons Attribution License, which permits unrestricted use, distribution, and reproduction in any medium, provided the original author and source are credited. 